\title{
Migration of Human Dendritic Cells in vitro Induced by Vaccines Stimulating Humoral and Cell Immunity
}

\author{
DOI: 10.17691/stm2016.8.3.10
}

Received February 9, 2016

V.Y. Talayev, MD, DSc, Professor, Head of Cellular Immunology Laboratory'; Professor,

Department of Microbiology and Immunology2;

M.V. Talaeva, PhD, Senior Researcher, Cellular Immunology Laboratory';

E.V. Voronina, Junior Researcher, Cellular Immunology Laboratory';

O.N. Babaykina, MD, PhD, Senior Researcher, Cellular Immunology Laboratory ${ }^{1}$

${ }^{1}$ Nizhny Novgorod Scientific Research Institute of Epidemiology and Microbiology named after academician I.N. Blokhina, 71 M. Yamskaya St., Nizhny Novgorod, 603950, Russian Federation;

${ }^{2}$ Nizhny Novgorod State Medical Academy, 10/1 Minin and Pozharsky Square, Nizhny Novgorod, 603005, Russian Federation

Dendritic cells (DC) are specialized antigen-presenting cells. One of their function is to deliver antigens from peripheral tissues to lymphoid organs by migration controlled by chemokines.

The aim of the investigation was to study the effect of vaccines stimulating cellular or humoral response on the expression of chemokine receptors CCR7, CXCR4 and CXCR5 on DC, and assess the motility of the cells and migration response on chemokines.

Materials and Methods. Immature DC derived from monocytes in vitro were incubated with vaccines. We used tuberculosis vaccine BCG stimulating, primarily, cellular response in vivo; hepatitis B vaccine stimulating antibody production, as well as aluminium hydroxide adjuvant. Reference DC maturation was induced with a cocktail of inflammatory mediators. Then, we assessed DC maturation and studied CCR7 gene expression, the presence of CCR7, CXCR4 and CXCR5 receptors on the outer membrane, spontaneous cell motility and chemotaxis induced by chemokines CCL21 and CXCL13.

Results. BCG effectively induces DC maturation, has no effect on the expression of receptors CXCR4 and CXCR5 and causes slight but reliable enhanced expression of gene and receptor CCR7 as well as CCL21 induced chemotaxis. Hepatitis B vaccine causes partial DC maturation and increases significantly the expression of receptors CCR7, CXCR4 and CXCR5, but does not increase spontaneous cell motility and enhances weakly chemotaxis in response to CCL21 and CXCL13.

Conclusion. Tuberculosis vaccine and hepatitis B vaccine induce different sets of chemokine receptors on DC, however, they stimulate DC hemotaxis relatively weakly. The findings suggest the necessity of searching new adjuvants, which enable to enhance the migration of DC carrying antigens to lymphoid organs.

Key words: dendritic cells; chemokine receptors; migration of dendritic cells; vaccines.

Models of immune responses in vitro are used both in fundamental and applied researches to study molecular mechanisms of the effect of drugs, and the development of new pharmaceuticals with a direct effect on the functions of immune cells and the ways of cellbased therapy. The present study used in vitro research techniques to assess the effect of two extensively used vaccines (tuberculosis and hepatitis $B$ ) on migration characteristics of human dendritic cells (DC).

DC are specialized antigen presenting cells with unique ability to involve naïve $\mathrm{T}$ cells in adaptive immune responses [1]. At the stage of the so called immature DC (iDC) these cells can effectively collect antigens by endocytosis. In case of infection, iDC capture microbial antigens and recognize pathogen-associated molecular patterns as well as endogenous inflammatory mediators: proinflammatory cytokines, alarmins and prostaglandin $E_{2}\left(P_{G}\right)[2,3]$. The recognition of these molecules leads to DC maturation resulting in the attenuation of endocytosis and upregulation of molecules important for $\mathrm{T}$ cell priming. Mature DC leave peripheral tissue and migrate through afferent lymphatic vessels into draining lymph nodes (LN). In LN, naïve $\mathrm{CD}^{+} \mathrm{T}$ cells recognize specific antigens on DC, proliferate and differentiate into various T-helper (Th) types with unique cytokine profiles and different functions to launch adaptive immune reactions. Therefore, one of the central functions of DC is the transport of antigens into lymphoid organs for immune response induction.

During the preparation for migration, the number of receptors for chemokines of peripheral tissues on DC membrane decreases, and instead of them there being expressed receptors of chemokines guiding the cells in regional LN. Among these newly expressed receptors, the key role is assigned to CCR7, ligands of which are produced throughout a migration path of DC

For contacts: Vladimir Y. Talayev, e-mail: talaev@inbox.ru 
from the peripheral tissue to T cell zone of LN $[4,5]$. So, at a starting point of the path, one of these ligands chemokine CCL21 - is produced in the endothelium of initial sections of lymph capillaries engaging migrating DC in lymphatic vessels $[5,6]$. When entering a draining $L N$ with a lymph flow, DC keep moving under the gradient of CCL19 and CCL21, powerful production of which occurs in paracortex - the end point of the route [2, 5-7]. In skin, the role of a supplementary chemo-attractant of $D C$ in a lymph stream is played by chemokine CXCL12 recognized by CXCR4 receptor [8].

However, the main migration route of DC in LN is not the only one. A part of DC of derma and marginal area of the spleen was shown to express chemokine receptor CXCR5 [9]. The receptor and its ligand CXCL13 play a key role in the migration of B-lymphocytes and T-follicular helpers (Tfh) in LN perifollicular areas and follicles: the induction zone of a humoral immune response [10], where CXCL13 producers are concentrated [11, 12]. Skin CXCR5 ${ }^{+}$DC respond to $\mathrm{CXCL} 13$ and migrate in B-cell area of LN at adaptive transfer [9]. A functional role of such DC migration is proved in the system with a selective inhibition of CXCR5 gene expression in DC, T-lymphocytes and B-cells in Heligmosomoides polygyrus-infested mice [13]. Recently, we have found that vaccines stimulating a humoral response are also able to induce CXCR5 expression on DC derived from monocytes in vitro [14].

The aim of the investigation was to study the effect of tuberculosis vaccine BCG, stimulating, primarily, cellular response; hepatitis $B$ vaccine stimulating antibody production, as well as aluminium hydroxide adjuvant on the expression and distribution of chemokine receptors CCR7, CXCR4 and CXCR5 on dendritic cells; assess the motility of the cells treated with vaccines, and their migration response on corresponding chemokines.

Materials and Methods. In the study we used tuberculosis vaccine BCG (Microgene, Russia), and yeast recombinant hepatitis B vaccine (Combiotech, Russia), as well as a suspension of aluminium hydroxide (AH) gel (Combiotech, Russia). A dose of BCG vaccine contains $50 \mu \mathrm{g}$ of lyophilized Calmette-Guérin bacilli ((2-4) $10^{7}$ living bacteria). A dose of hepatitis $B$ vaccine contains $10 \mu \mathrm{g}$ of recombinant HBs-antigen sorbed on $\mathrm{AH}$ gel $\left(250 \mu \mathrm{g} \mathrm{A}^{3+}\right)$. Before using hepatitis $\mathrm{B}$ vaccine and $\mathrm{AH}$ gel were washed them free from merthiolate by RPMI-1640 medium (Gibco, Great Britain) by means of centrifugation.

DC were derived from venous blood monocytes of healthy adult donors. For this purpose blood mononuclear cells were isolated by centrifugation above the Hystopaque-1077 layer (Sigma-Aldrich, USA), inoculated in 24-well plates (Costar, USA) by $5 \cdot 10^{6}$ cell per a well, and incubated at $37^{\circ} \mathrm{C}$ and $5 \% \mathrm{CO}_{2}$. After $2 \mathrm{~h}$, non-adherent cells were removed, and adherent ones were cultivated in RPMI-1640 medium with 10\% fetal calf serum (FCS) (PAA Laboratories, Austria),
$20 \mathrm{ng} / \mathrm{ml}$ interleukin-4 (IL-4) (R\&D, USA) and $100 \mathrm{ng} /$ $\mathrm{ml}$ granulocyte-macrophage colony-stimulating factor (GM-CSF) (R\&D, USA). IL-4 and GM-CSF were added repeatedly in cultures up to the same concentration on culture day 3 . On day $7 \mathrm{iDC}$ derived from monocytes were stimulated by vaccines at the concentration being 0.2 and $0.02 \mathrm{dose} / \mathrm{ml}$. AH gel suspension was used at equivalent concentrations ( 50 and $5 \mu \mathrm{g} / \mathrm{ml} \mathrm{Al}^{3+}$ ).

The development of control mature DC was induced by a mixture of inflammatory mediators consisting of 25 $\mathrm{ng} / \mathrm{ml} \mathrm{IL-1 \beta ,} 25 \mathrm{ng} / \mathrm{ml} \mathrm{IL}-6,50 \mathrm{ng} / \mathrm{ml}$ of tumor necrosis factor- $\alpha$ (R\&D, USA) and $1 \mu \mathrm{g} / \mathrm{ml} \quad \mathrm{PGE}_{2}$ (SigmaAldrich, USA). $48 \mathrm{~h}$ after stimulation the cells were collected, separated from hepatitis B vaccine or $\mathrm{AH}$ gel by centrifugation above $85 \%$ percoll layer (SigmaAldrich, USA), and used to analyze a phenotype, migration characteristics and RNA extraction for reverse transcription and polymerase chain reaction (RT-PCR).

A cell phenotype was determined using laser flow cytometry of DC labeled by fluorescent antibody conjugates to molecules HLA-DR, CD14 (Sorbent, Russia), CD80, CD83, CD86, CCR7, CXCR4 and CXCR5 (BD Biosciences, USA). The samples were studied using flow cytofluorometer FacsCalibur (BD Biosciences, USA) gating DC according to forward and side scatter profile.

DC migration was measured by chemotaxis through a polycarbonate filter with pore size $8 \mu \mathrm{m}$ in 24-well chambers ThinCerts (Greiner bio-one, Switzerland). For this effect, $10^{5}$ cells washed free from FCS and resuspended in RPMI-1640 were placed into an upper chamber. To induce chemokine-dependent chemotaxis, chemokines CCL21 or CXCL13 (R\&D, USA) diluted in RPMI-1640 without FCS were placed in a well (a lower chamber) at the concentrations of $500 \mathrm{ng} / \mathrm{ml}$ and $1 \mu \mathrm{g} /$ $\mathrm{ml}$, respectively. For controls we used lower chambers with a medium without chemokines. After 2-hour incubation at $37^{\circ} \mathrm{C}$ and $5 \% \mathrm{CO}_{2}$ migratory $\mathrm{DC}$ were collected from the lower chambers and counted. DC mobility was determined in other experiments after 19hour incubation. In these experiments upper and lower chambers of ThinCerts contained RPMI-1640 with 10\% FCS without chemokines.

To assess CCR7 gene expression, we extracted RNA from $10^{5}$ cells using NucleoSpinRNA XS (MachereyNagel, Germany) and estimated the number of transcripts at one-stage relative quantitative RT-PCR in real-time mode using Stratagene Mx3005P (Agilent Technologies, USA) with TaqMan One-Step RT-PCR Master Mix Reagents kit, primers and labeled samples TaqMan gene expression assay reagents in order to determine CCR7 gene expression (Hs01013469_m1) and 32 -microglobulin (Applied Biosystems, USA). The measurements were made in triplicates. The diagrams were equalized according to a normalization stain. Ct value (amplification threshold cycle) was determined as maximum of the second order derivative of DNA 
cumulative function. Normalization was performed according to $\beta 2$-microglobulin gene expression level. iDC samples were used as a calibrator.

Cytometric findings were processed using CellQuest program estimating the percentage of cells carrying a marker, and the marker expression density by a geometrical mean of fluorescence intensity (GMFI) of the stained cells. PCR findings were processed using Stratagene Mx3005P. Statistically the data were analyzed by Student t-test.

Results. For experiments we used DC derived from monocytes of healthy adult donors by culturing with IL-4 and GM-CSF. During the culture with these cytokines, the cells lost their monocyte marker CD14, enhanced the expression density (GMFI) of major histocompatibility complex molecules HLA-DR, and acquired the expression of co-stimulating molecules CD80 and CD86.
As a result, the obtained cells had typical iDC phenotype: $\mathrm{CD}_{14}-\mathrm{HLA}-\mathrm{DR}{ }^{+} \mathrm{CD} 80^{+} \mathrm{CD} 83^{\text {low/-CD86 }}{ }^{+}$(Figure 1). Stimulation of iDC by a mixture of proinflammatory cytokines and $\mathrm{PGE}_{2}$ induced cell maturation, which was evident in the expression of a marker of mature $D C$ CD83 and additional increase of HLA-DR, CD80 and CD86 expression levels. Such cytokine-stimulated cells (DC-CTK) with a phenotype of typical mature DC were used as positive mature control, and non-stimulated iDC were used as negative controls.

BCG vaccines added in iDC cultures at the final concentration 0.2 dose $/ \mathrm{ml}$ induced maturation of cells with a phenotype, which was nearly similar to that of DC-CTK (See Figure 1). Hepatitis B vaccine at the concentration 0.2 dose $/ \mathrm{ml}$ and its adjuvant component (AH gel) also increased the expression of HLA-DR, CD83 and CD86 molecules, however, the expression

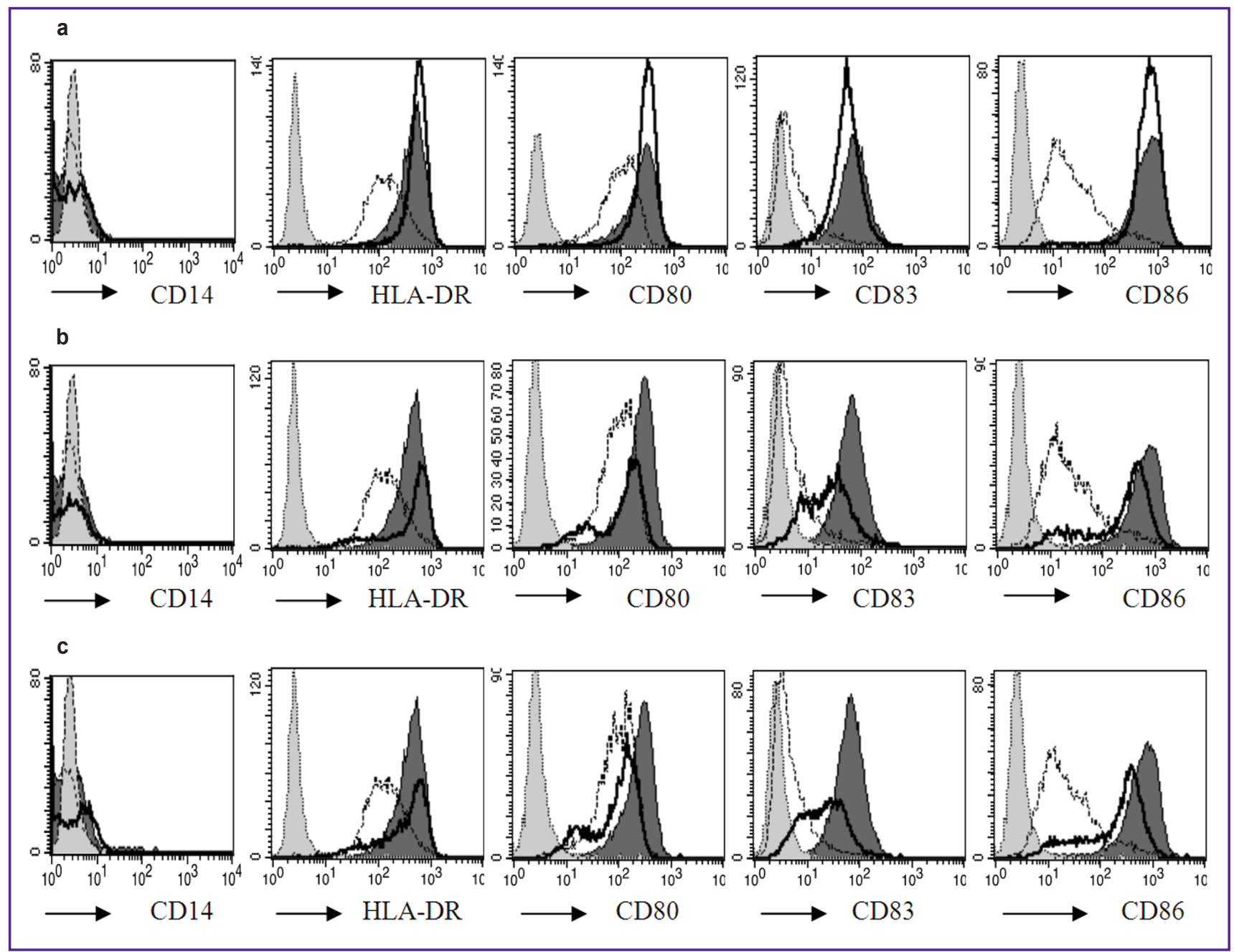

Figure 1. CD14, HLA-DR, CD80, CD83 and CD86 expression on dendritic cells after BCG (a), hepatitis B (b) vaccine, and $\mathrm{AH}$ gel suspension (c) stimulation is marked by a black line. A thin dotted line shows the expression of the same molecules on iDC, and on DC-CTK is indicated by a histogram with a dark grey field. Light grey histogram with a dotted line shows isotype control. Concentration of vaccines is $0.2 \mathrm{dose} / \mathrm{ml}$, and that of aluminium hydroxide is $50 \mu \mathrm{g} / \mathrm{ml} \mathrm{Al}{ }^{3+}$. The result of representative experiment $(n=15)$. $Y$ axis shows the number of events 
density was not high, and the phenotype of the cells corresponded to the so called semi-mature DC.

iDC maturation into mature DC was accompanied by the expression of chemokine receptors able to guide
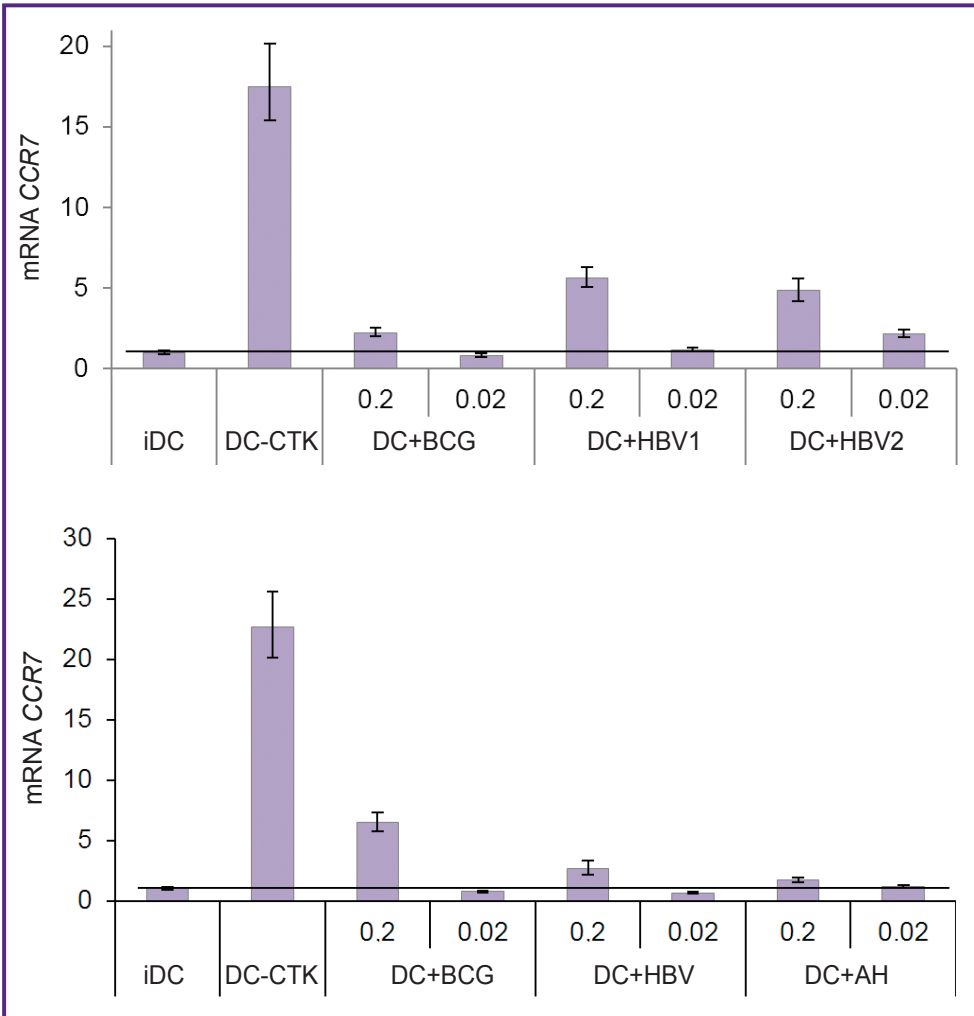

Figure 2. CCR7 gene expression in dendritic cells. The cells incubated with $B C G$ vaccine are marked by $D C+B C G$, with samples of hepatitis $B$ vaccine - by DC+HBV, DC+HBV1, DC+HBV2, with $\mathrm{AH}$ gel by $\mathrm{DC}+\mathrm{AH}$. The results of two representative RT-PCR contain five figures. The numbers above the types of dendritic cells indicate the concentration of vaccines (dose $/ \mathrm{ml})$ cell migration in regional lymph nodes. So, mature DCCTK showed high expression of mRNA of CCR7 gene encoding a key receptor for DC migration from peripheral tissues to T-cell zones of draining LN (Figure 2). DC stimulated by BCG vaccine and hepatitis $B$ vaccine at the concentration of 0.2 dose/ $\mathrm{ml}$ or $\mathrm{AH}$ gel at an equivalent concentration also induced this gene expression, however, the abundance of its transcripts in cells was significantly lower than DC-CTK level. The vaccines used at a final concentration 0.02 dose/ml resulted in no significant CCR7 gene expression growth.

Immature DC had a very low expression level of CCR7 protein. CCR7 gene expression growth at DC maturation resulted in significant enhancement of CCR7 receptor expression on an outer cell membrane. DC-CTK and DC after hepatitis $B$ vaccine stimulation were found to have the highest expression level of CCR7 receptor (Figure 3). DC incubated with $\mathrm{AH}$ gel particles had significantly lower expression of CCR7 protein than DC-CTK $(p=0.01)$ and DC stimulated by hepatitis $B$ vaccine $(p=0.002)$. BCG stimulated $D C$ also demonstrated a lower increase in CCR7 expression on a membrane compared to DCCTK $(p=0.01)$ and DC stimulated by hepatitis $B$ vaccine $(p=0.04)$.

The study of other chemokine receptors able to have an effect migration route of mature DC in lymphoid organs showed the incubation of cells with hepatitis $B$ vaccine or $\mathrm{AH}$ gel to induce marked expression of CXCR5 receptor on DC (See Figure 3). DCCTK maturation leads to the appearance of

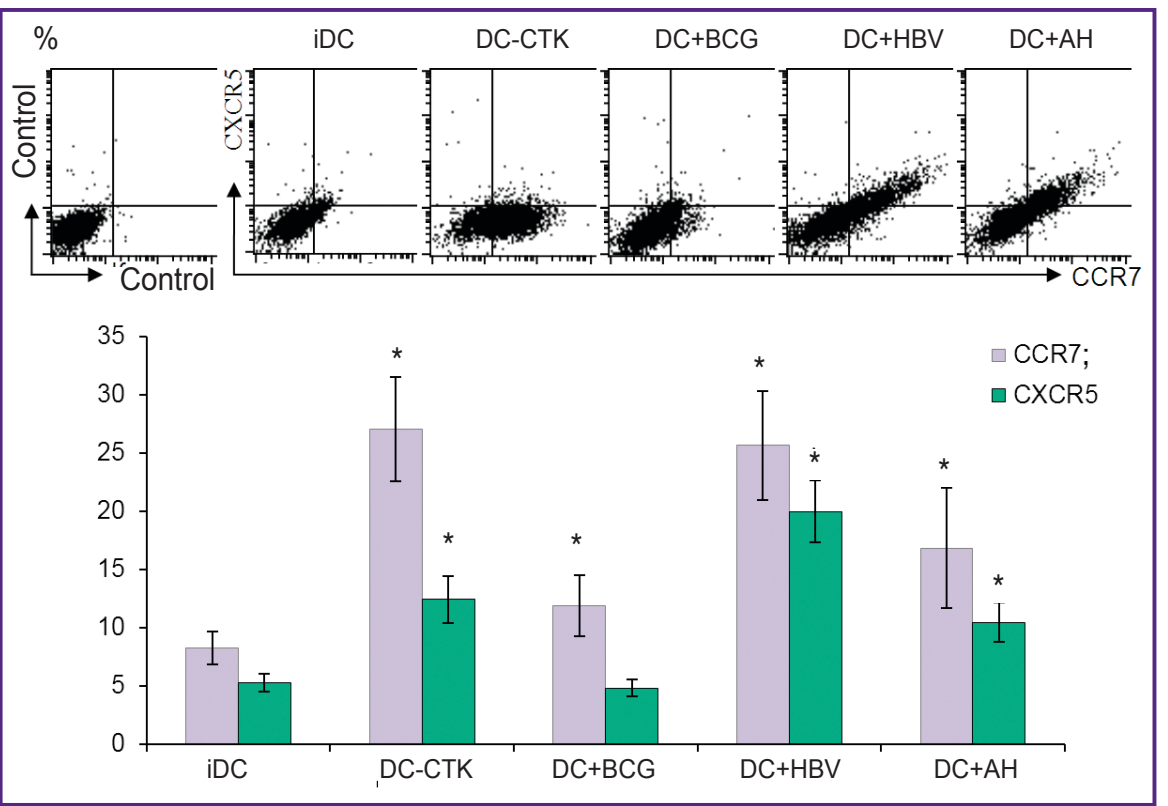

Figure 3. CCR7 and CXCR5 expression on an outer membrane of dendritic cells after the incubation with vaccines. Above: the result of a cytometric analysis of the distribution of chemokine receptors on cells. Below: average percentage composition of $\mathrm{CCR}^{+}$and $\mathrm{CXCR}^{+}$DC $(\mathrm{n}=19)$. * Significant differences of values with iDC in a pair t-test $(p<0.05)$. Cell types are indicated similar to those used in Figure 2 
CXCR5 $5^{+}$cells but these cells have a small number of CXCR5 receptors on a membrane that is evident as a low increase of GMFI. Control iDC as well as BCG stimulated DC actually had no CXCR5 on their surfaces. A distribution analysis of chemokine receptors on cells revealed the co-expression of CXCR5 and CCR7 on hepatitis B or AH gel stimulated DC (See Figure 3).

In contrast to CCR7 and CXCR5, chemokine receptor CXCR4 is present on a larger part of iDC (Figure 4). Stimulation by inflammatory mediators, hepatitis B or $\mathrm{AH}$ gel induces additional expansion in the number of CXCR4 ${ }^{+}$cells (Figure 4 (b)), and results in fluorescence intensity growth (Figure 4 (c)). Maximal GMFI was found on CXCR4 ${ }^{+} \mathrm{DC}$ after incubation with hepatitis $B$ vaccine or AH. CCR7 and CXCR5 under these conditions are expressed, primarily, on DC with a high level of
CXCR4 expression (Figure 4 (d)). BCG vaccine has no significant effect on CXCR4 expression.

The function of CCR7 and CXCR5 receptors was assessed by chemotaxis induced by the corresponding chemokines. The study involved a two-hour test of cells migrating from a medium-containing chamber through polycarbonate filter pores into a chamber with chemokine solution. Despite the fact that the test as a control ones implies spontaneous cell mobility determination (chemokine-free migration), for an accurate assessment of cell mobility we additionally determined spontaneous DC mobility in a 19-hour test. DC-CTK were found to have both high spontaneous mobility as well as marked capability to targeted CCR7dependent chemotaxis. If chemokines were absent, these cells moved actively over the filter surface, and as
Figure 4. CXCR4 expression on dendritic cells: (a) a representative cytometric CXCR4 analysis on DC after BCG, hepatitis B vaccines and $\mathrm{AH}$ gel stimulation (a thick line). CXCR4 expression is also shown on iDC (a thin dotted line) and DCCTK (a histogram with a dark grey field). A light grey histogram with a dotted line indicates isotype control. Diagrams (b) and (c) show average percentage composition of CXCR4+ dendritic cells of adults and GMFI, respectively $(n=17) ; \quad$ * significant differences of values with iDC in a pair t-test $(p<0.05) ;(d)$ is the distribution of chemokine receptors on dendritic cells. Gate R2 marks the cells with high CXCR4 expression. Cell types are indicated similar to those used in Figure 2

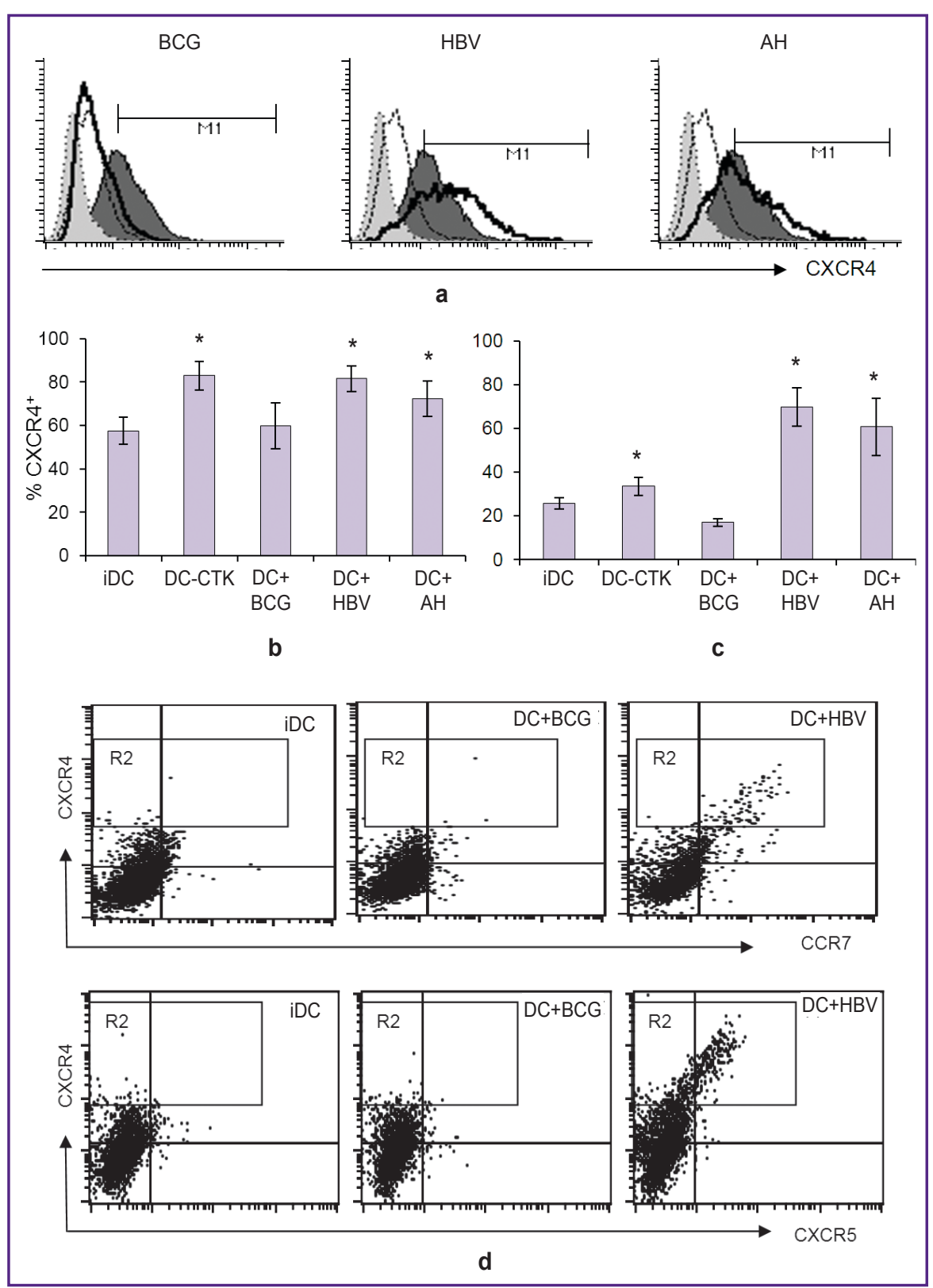


a result, nearly one third of them within $19 \mathrm{~h}$ succeeded in filtering through the pores into the lower chamber (Figure 5 (c)). In two-hour test 1.5\% DC-CTK migrated through a filter without chemokines. Chemokine CCL21 (ligand CCR7) significantly enhanced the migration of these cells, and during 2-hour incubation, $12.8 \%$ cells brought into the system managed to migrate in a chamber with chemokine (Figure $5(\mathrm{a})$ ). The number of migrated cells corresponds to $47.3 \%$ of $\mathrm{CCR} 7^{+} \mathrm{DC}$, i.e., of all the cells capable of CCL21 dependent chemotaxis (See Figure 3).

BCG vaccine stimulation had no significant effect on spontaneous DC mobility, but increased CCR7dependent chemotaxis, and $4.5 \%$ cells migrated in a chamber with chemokine during $2 \mathrm{~h}$. Chemotaxis increase was significant ( $p=0.015$ compared to iDC), but was remarkably lower than the correspondent index of DC-CTK $(p=0.025)$. Considering a low content of $C C R 7^{+}$ DC in the cultures incubated with BCG, the number of migrated cells corresponds to $37.8 \%$ of all $\mathrm{CCR} 7^{+} \mathrm{DC}$ of these cultures.

The stimulation of DC by hepatitis $B$ vaccine at the concentration of 0.2 dose $/ \mathrm{ml}$ induces a low increase of CCR7-dependent migration (Figure 5 (a)), despite a marked CCR7 expression on stimulated cells (Figure 3 (b)). Within $2 \mathrm{~h}$, less than 2\% DC brought and only $7.7 \%$ $\mathrm{CCR}^{+}$cells potentially capable to such chemotaxis migrate in response to chemokine. The unexpected result can be due to the low mobility of cells taken up a great number of vaccine particles. Actually, these vaccine-stimulated DC show lower mobility in a 19-hour test compared to all DC types including iDC (See Figure 5 (c)). A tenfold decrease of vaccine concentration restores cell mobility up to iDC level, but cancels an effect on CCR7 expression (See Figure 2). The same reason, i.e. low mobility, is likely to prevent hepatitis $B$ vaccine-bearing $D C$ from effective migration in response to $\mathrm{CXCL} 13$ - a chemokine recognized by CXCR5 receptor. As mentioned above, this vaccine induces significant CXCR5 expression on a cell membrane (See Figure 3), but a migration test showed just a slight increase of CXCR5-dependent chemotaxis, and only $2.1 \%$ of added DC incubated with 0.2 dose $/ \mathrm{ml}$ vaccine had time to migrate within $2 \mathrm{~h}$ responding to CXCL13 (Figure 5 (b)). Despite a small scale, the increase of CXCR5-dependent chemotaxis under hepatitis

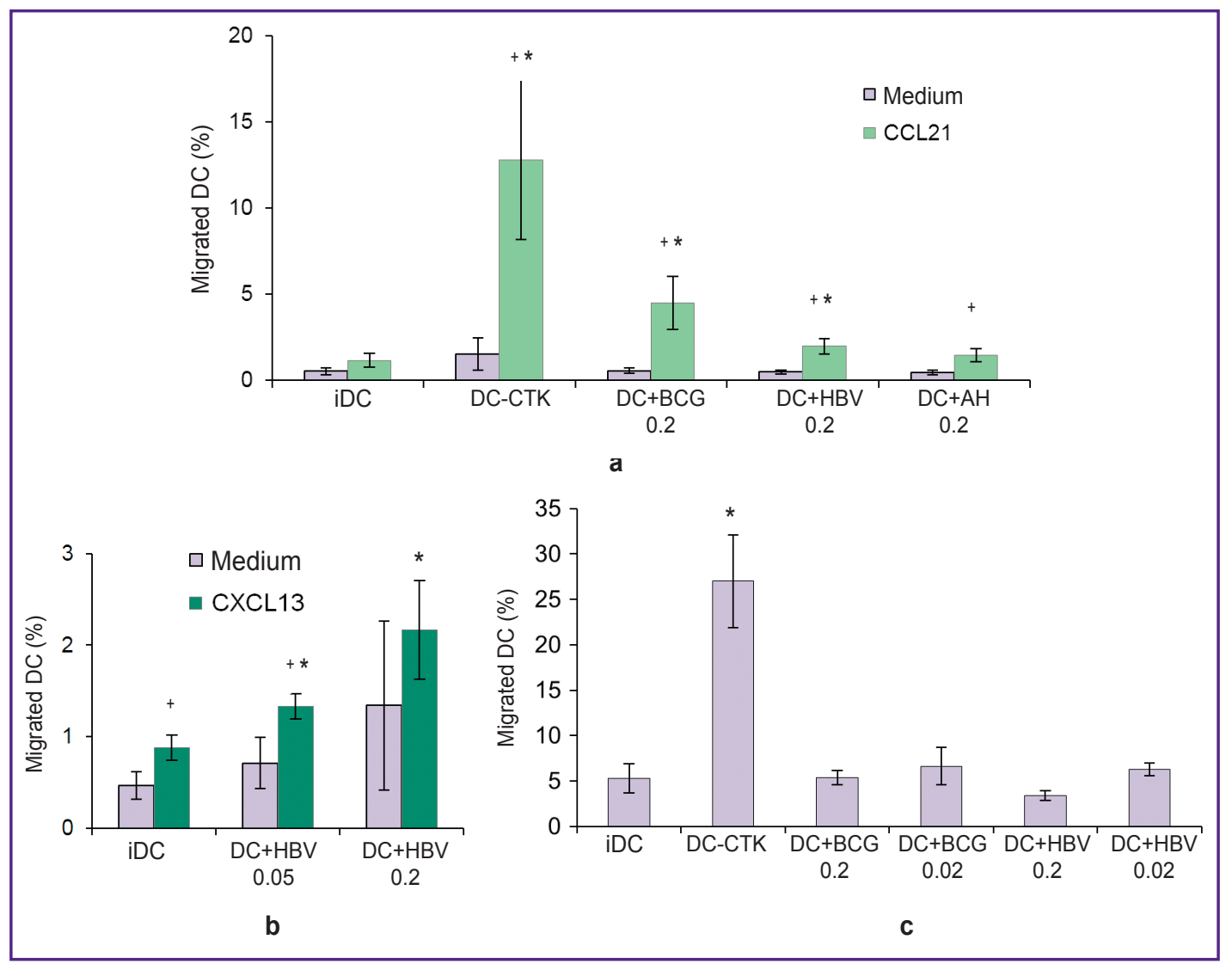

Figure 5. Chemokine-induced and spontaneous migration of dendritid cells: (a) a migration response of the cells to CCL21 $(n=10)$; (b) a migration response to $\operatorname{CXCL13~(n=5);~(c)~spontaneous~migration~of~the~cells~within~} 19 \mathrm{~h}(\mathrm{n}=7)$; ${ }^{*}$ significant differences of values with iDC in a pair t-test $(p<0.05) ;{ }^{+}$significant differences of chemokine-induced chemotaxis with spontaneous migration of the corresponding cell type in a pair $t$-test $(p<0.05)$. The symbols indicating the cell type are similar to those used in Figure 2, the concentration of vaccines (dose $/ \mathrm{ml})$ is shown near the indicated cell types 
$B$ vaccine is of statistical significance (for vaccine concentration 0.05 dose $/ \mathrm{ml} p=0.013$, for concentration 0.2 dose $/ \mathrm{ml} p=0.045$ ).

Discussion. The in vitro study of the vaccines' effect on DC maturation and migration showed BCG vaccine to induce effectively phenotypic $D C$ maturation and cause a moderate increase CCR7 gene and receptor expression. This chemokine receptor is typical for mature DC and necessary for DC migration from peripheral tissues (e.g. from the site of vaccine injection) in lymphatic vessels, and further - in paracortex, i.e. in T-cell zone of LN. As was shown before [15], BCG effectively increases DC ability to stimulate type 1 T-helpers (Tx1) essential to the protection against tuberculosis. In this regard, CCR7-mediated delivery of the vaccine antigens to T-cell zone of LN seems to be more beneficial for the meeting with naïve $\mathrm{CD}^{+}{ }^{+}$-lymphocytes and guiding them for maturation in $\mathrm{Tx} 1$, since an inductive phase of the response is localized in T-cell zone of lymphoid organs. However, the analysis of CCR7 expression and its function (chemotaxis) demonstrates a relatively low mobilizing effect of BCG on CCR7-dependent DC migration compared to the level of control mature DC. In our opinion, the weak stimulation of DC chemotaxis by BCG vaccine may be a factor limiting the vaccine efficiency. Moreover, BCG vaccine in contrast to the mixture of inflammatory mediators does not increase spontaneous DC mobility, which can contribute to cells leaving peripheral tissues and active search of contacts with T-lymphocytes in LN parenchyma on the first day after migration [16].

Hepatitis $B$ vaccine and its adjuvant component $\mathrm{AH}$ induce partial phenotypic DC maturation accompanied by the increase in chemokine receptor CXCR4 expression and induction of CCR7 and CXCR5 expression on a considerable part of cells. It is our opinion that this expression type of chemokine receptors should promote the delay of at least some part of migrating $\mathrm{DC}$ in the outer layer of $\mathrm{LN}$ cortex, where chemokine CXCL13 is produced in follicles [11, 12]. In addition, in human, in a mantle area of follicles and interfollicular region [17], and in mice - in a dark area of follicles [18] chemokine CXCL12 is produced, which can also contribute to the delay of DC with high CXCR4 expression level. Moreover, CXCR5 expression on DC, B-cells and maturating T-follicular helpers can result in co-localization of all potential participants of humoral immune response to vaccine near or inside B-cell follicles promoting the humoral immune response development. In this regard, such delivery route of antigens seems to be favorable for vaccine stimulating antibody production; however, the disadvantage of hepatitis $B$ vaccine is a low mobilizing effect on DC migration that must be due to low mobility of the cells after absorption of a large amount of vaccine sorbed on $\mathrm{AH}$ gel particles.

Conclusion. Tuberculosis vaccine and hepatitis $B$ vaccine induce different sets of chemokine receptors on dendritic cells, however, they relatively weakly mobilize the cells for migration in lymphoid organs. The findings suggest the necessity of searching new adjuvant components of vaccines able to enhance the trafficking of dendritic cells in T- and B-cell zones of lymphoid organs improving the efficiency of antigen delivery in the areas of immune response induction.

Study Funding. The study was carried out as a part of a sectoral research program 2011-2015 "Scientific researches and studies in order to provide sanitary and epidemiological welfare and the decrease of incidence of infectious diseases in Russian Federation".

Conflicts of Interest. The authors declare no conflicts of interest related to the present study.

\section{References}

1. Steinman R.M. The dendritic cell system and its role in immunogenicity. Annu Rev Immunol 1991; 9: 271-296, http:// dx.doi.org/10.1146/annurev.immunol.9.1.271.

2. Alvarez D., Vollmann E.H., von Andrian U.H. Mechanisms and consequences of dendritic cell migration. Immunity 2008; 29(3): 325-342, http://dx.doi.org/10.1016/j. immuni.2008.08.006.

3. Talaev V.Yu. The mechanisms controlling migration of myeloid dendritic cells and langerhans cells. Immunologiya 33(2): 104-112.

4. Ohl L., Mohaupt M., Czeloth N., Hintzen G., Kiafard Z., Zwirner J., Blankenstein T., Henning G., Förster R. CCR7 govern skin dendritic cell migration under inflammatory and steady-state conditions. Immunity 2004; 21(2): 279-288, http:// dx.doi.org/10.1016/j.immuni.2004.06.014.

5. Johnson L.A., Jackson D.G. Cell traffic and the lymphatic endothelium. Ann N Y Acad Sci 2008; 1131: 119133, http://dx.doi.org/10.1196/annals.1413.011.

6. Randolph G.J., Angeli V., Swartz M.A. Dendritic-cell trafficking to lymph node through lymphatic vessels. Nat Rev Immunol 2005; 5(8): 617-628, http://dx.doi.org/10.1038/ nri1670.

7. Martín-Fontecha A., Sebastiani S., Höpken U.E., Uguccioni M., Lipp M., Lanzavecchia A., Sallusto F. Regulation of dendritic cell migration to the draining lymph node. Impact on T lymphocyte traffic and priming. J Exp Med 2003; 198(4): 615-621, http://dx.doi.org/10.1084/jem.20030448.

8. Kabashima K., Shiraishi N., Sugita K., Mori T., Onoue A., Kobayashi M., Sakabe J., Yoshiki R., Tamamura H., Fujii N., Inaba K., Tokura Y. CXCL12-CXCR4 engagement is required for migration of cutaneous dendritic cells. Am J Pathol 2007; 171(4): 1249-1257, http://dx.doi.org/10.2353/ ajpath.2007.070225.

9. Saeki H., Wu M.T., Olasz E., Hwang S.T. A migratory population of skin-derived dendritic cells expresses CXCR5, responds to $B$ lymphocyte chemoattractant in vitro, and colocalizes to B cell zones in lymph nodes in vivo. Eur J Immunol 2000; 30(10): 2808-2814, http://dx.doi.org/10.1002/15214141(200010)30:10<2808::aid-immu2808>3.0.co;2-k.

10. Toptygina A.P. The lymphoid follicle - the immune response zone. Immunologiya 2012; 33(3): 162-168.

11. Cyster J.G., Ansel K.M., Reif K., Ekland E.H., Hyman P.L., Tang H.L., Luther S.A., Ngo V.N. Follicular stromal cells and lymphocyte homing to follicles. Immunol 
Rev 2000; 176(1): 181-193, http://dx.doi.org/10.1034/j.1600065x.2000.00618.x.

12. Katakai T., Suto H., Sugai M., Gonda H., Togawa A., Suematsu S., Ebisuno Y., Katagiri K., Kinashi T., Shimizu A. Organizer-like reticular stromal cell layer common to adult secondary lymphoid organs. J Immunol 2008; 181(9): 61896200, http://dx.doi.org/10.4049/jimmunol.181.9.6189.

13. León B., Ballesteros-Tato A., Browning J.L., Dunn R., Randall T.D., Lund F.E. Regulation of $\mathrm{T}_{\mathrm{H}} 2$ development by $\mathrm{CXCR}^{+}$dendritic cells and lymphotoxin-expressing B cells. Nat Immunol 2012; 13(7): 681-690, http://dx.doi.org/10.1038/ni.2309.

14. Talayev V.Yu., Plehanova M.V., Zaichenko I.Ye., Babaykina O.N. Effect of vaccines on the expression of chemokine receptors on dendritic cells of newborns and adults in vitro. Immunologiya 2013; 34(6): 318-323.

15. Plehanova M.V., Talayev V.Yu., Babaykina O.N.,
Zaichenko I.Ye., Ephimov E.I. The action of BCG and hepatitis B vaccines on phenotypic and functional properties of the newborn's dendritic cells in vitro. Immunologiya 2012; 33(6): 311-318.

16. Lindquist R.L., Shakhar G., Dudziak D., Wardemann H., Eisenreich T., Dustin M.L., Nussenzweig M.C. Visualizing dendritic cell networks in vivo. Nat Immunol 2004; 5(12): 12431250, http://dx.doi.org/10.1038/ni1139.

17. Wang C., Hillsamer P., Kim C.H. Phenotype, effector function, and tissue localization of PD-1-expressing human follicular helper T cell subsets. BMC Immunology 2011; 12: 53, http://dx.doi.org/10.1186/1471-2172-12-53.

18. Allen C.D., Ansel K.M., Low C., Lesley R., Tamamura H., Fujii N., Cyster J.G. Germinal center dark and light zone organization is mediated by CXCR4 and CXCR5. Nat Immunol 2004; 5(9): 943-952, http://dx.doi.org/10.1038/ni1100. 